\title{
Condensate Fraction of a Two-Dimensional Attractive Fermi Gas
}

\author{
Luca Salasnich \\ CNR-INFM and CNISM, Dipartimento di Fisica "Galileo Galilei", \\ Università di Padova, Via Marzolo 8, 35131 Padova, Italy
}

(Dated: November 1, 2018)

\begin{abstract}
We investigate the Bose-Einstein condensation of fermionic pairs in a two-dimensional uniform two-component Fermi superfluid obtaining an explicit formula for the condensate density as a function of the chemical potential and the energy gap. By using the mean-field extended BCS theory, we analyze, as a function of the bound-state energy, the off-diagonal long-range order in the crossover from the Bardeen-Cooper-Schrieffer (BCS) state of weakly-bound Cooper pairs to the Bose-Einstein Condensate (BEC) of strongly-bound molecular dimers.
\end{abstract}

PACS numbers: 03.75.Hh, 03.75.Ss

In the last few years several experimental groups have observed the crossover from the Bardeen-CooperSchrieffer (BCS) state of Cooper Fermi pairs to the BoseEinstein condensate (BEC) of molecular dimers with ultra-cold two hyperfine component Fermi vapors of ${ }^{40} \mathrm{~K}$ atoms [1, 2, 3] and ${ }^{6} \mathrm{Li}$ atoms [4, 5]. A finite condensed fraction has been detected also in the BCS side of the crossover [2, 4], stimulating a debate over its interpretation 6, 7, 8, 9]. Extended BCS (EBCS) equations 10, 11, 12 have been used to reproduce density profiles 13] and collective oscillations [14] of these Fermi gases. Improvements based on Monte Carlo fitting and superfluid dynamics have been considered [15, 16], showing that EBCS is quite accurate. As this EBCS mean-field theory is defined for any value of the coupling, it provides an interpolation between the BCS weak-coupling regime and the BEC strong-coupling limit 17, 18]. Despite well know limitations [17] the EBCS theory is considered a reliable approximation for studying the whole BCS-BEC crossover at zero temperature, giving a simple and coherent description of the crossover in terms of fermionic variables.

Recently, within the EBCS scheme, we have derived 19 an explicit formula for the number of condensed fermionic pairs in the uniform BCS ground-state. We have used the EBCS equations to study the behavior of this condensate fraction as a function of the interatomic scattering length in the BCS-BEC crossover: from the BCS regime crossing the unitarity limit to the BEC regime. With no fitted parameters, we have found a remarkable agreement with experimental results [4] indicating a relevant fraction of condensed pairs of ${ }^{6} \mathrm{Li}$ atoms also on the BCS side of the Feshbach resonance. Note that our analytic formula [19] of the condensed fraction of the three-dimensional (3D) attractive Fermi gas has been obtained independently also by Ortiz and Dukelsky [20] and Ohashi and Griffin [21]. The formula has been then compared with Monte Carlo calculations by Astrakharchik et al. [22]: these Monte Carlo results show that the analytical formula slightly overestimates the condensed fraction of Fermi pairs.

For repulsive Fermi gases, it has been predicted that a reduced dimensionality strongly modifies density pro- files [23, 24, 25, 26], collective modes [27] and stability of mixtures [28, 29]. In this paper we calculate the condensate fraction of Fermi pairs in a strictly 2D attractive Fermi system. It is well known that purely attractive potentials have bound states in $1 \mathrm{D}$ and $2 \mathrm{D}$ for any strength, contrary to the 3D case [30]. It follows that a bound state appears immediately as the two-body attraction is introduced in a 2D Fermi gas 31, 32]. As discussed by Marini, Pistolesi and Strinati [33], for a 2D Fermi superfluid in the EBCS theory it is the value of the bound-state energy that determines the crossover from BCS state of weakly-bound Cooper pairs to the BEC of strongly-bound dimers. We shall show that the condensate fraction of Fermi pairs, which can be expressed in terms of elementary functions, increases smoothly during the 2D BCS-BEC crossover and becomes equal to $1 / 2$ (all pairs are condensed) only for a very large bound-state energy.

The Hamiltonian density of a dilute and interacting two-spin-component Fermi gas in a box of volume $V$ is given by

$$
\hat{\mathcal{H}}=-\frac{\hbar^{2}}{2 m} \sum_{\sigma=\uparrow, \downarrow} \hat{\psi}_{\sigma}^{+} \nabla^{2} \hat{\psi}_{\sigma}+g \hat{\psi}_{\uparrow}^{+} \hat{\psi}_{\downarrow}^{+} \hat{\psi}_{\downarrow} \hat{\psi}_{\uparrow},
$$

where $\hat{\psi}_{\sigma}(\mathbf{r})$ is the field operator that destroys a Fermion of spin $\sigma$ in the position $\mathbf{r}$, while $\hat{\psi}_{\sigma}^{+}(\mathbf{r})$ creates a Fermion of spin $\sigma$ in $\mathbf{r}$. The attractive inter-atomic interaction is described by a contact pseudo-potential of strength $g$ $(g<0)$. The number density operator is

$$
\hat{n}(\mathbf{r})=\sum_{\sigma=\uparrow, \downarrow} \hat{\psi}_{\sigma}^{+}(\mathbf{r}) \hat{\psi}_{\sigma}(\mathbf{r})
$$

and the average number of fermions reads

$$
N=\int d^{3} \mathbf{r}\langle\hat{n}(\mathbf{r})\rangle .
$$

The interacting term can be treated within the meanfield Hartree-Fock approximation, namely

$$
\begin{aligned}
\hat{\psi}_{\uparrow}^{+} \hat{\psi}_{\downarrow}^{+} \hat{\psi}_{\downarrow} \hat{\psi}_{\uparrow} & =\left\langle\hat{\psi}_{\uparrow}^{+} \hat{\psi}_{\downarrow}^{+}\right\rangle \hat{\psi}_{\downarrow} \hat{\psi}_{\uparrow}+\hat{\psi}_{\uparrow}^{+} \hat{\psi}_{\downarrow}^{+}\left\langle\hat{\psi}_{\downarrow} \hat{\psi}_{\uparrow}\right\rangle \\
& +\left\langle\hat{\psi}_{\uparrow}^{+} \hat{\psi}_{\uparrow}\right\rangle \hat{\psi}_{\downarrow}^{+} \hat{\psi}_{\downarrow}+\hat{\psi}_{\uparrow}^{+} \hat{\psi}_{\uparrow}\left\langle\hat{\psi}_{\downarrow}^{+} \hat{\psi}_{\downarrow}\right\rangle
\end{aligned}
$$


and the Hamiltonian density (1) is diagonalized by using the following Bogoliubov representation of the field operator

$$
\hat{\psi}_{\uparrow}(\mathbf{r})=\frac{1}{V^{1 / 2}} \sum_{\mathbf{k}}\left(u_{k} e^{i \mathbf{k} \cdot \mathbf{r}} \hat{b}_{\mathbf{k} \uparrow}-v_{k} e^{-i \mathbf{k} \cdot \mathbf{r}} \hat{b}_{\mathbf{k} \downarrow}\right),
$$

in terms of the anticommuting quasi-particle Bogoliubov operators $\hat{b}_{\mathbf{k} \sigma}$. The quasi-particle amplitudes $u_{k}$ and $v_{k}$, such that $u_{k}^{2}+v_{k}^{2}=1$, are obtained by imposing the minimization [30] of the thermodynamic potential

$$
\Omega=\int d^{3} \mathbf{r}\langle\hat{\mathcal{H}}(\mathbf{r})-\mu \hat{n}(\mathbf{r})\rangle,
$$

where $\mu$ is the chemical potential, fixed by the average number $N$ of fermions. At zero-temperature the average of quasi-particle Bogoliubov operators is given by

$$
\left\langle\hat{b}_{\mathbf{k} \sigma}^{+} \hat{b}_{\mathbf{k} \sigma^{\prime}}\right\rangle=\Theta\left(E_{k}\right) \delta_{\sigma \sigma^{\prime}}
$$

where $E_{k}$ are the quasi-particle energies and $\Theta(x)$ is the Heaviside step function. Neglecting the Fock terms $\left\langle\hat{\psi}_{\sigma}^{+} \hat{\psi}_{\sigma^{\prime}}\right\rangle$ with $\sigma \neq \sigma^{\prime}[34]$, after minimization of (6) one recovers [35, 36] the standard BCS equation for the number of particles

$$
N=2 \sum_{\mathbf{k}} v_{k}^{2}
$$

and the familiar BCS gap equation

$$
-\frac{1}{g}=\frac{1}{V} \sum_{\mathbf{k}} \frac{1}{2 E_{k}}
$$

Here

$$
E_{k}=\left[\left(\frac{\hbar^{2} k^{2}}{2 m}-\mu\right)^{2}+\Delta^{2}\right]^{1 / 2}
$$

and

$$
v_{k}^{2}=\frac{1}{2}\left(1-\frac{\frac{\hbar^{2} k^{2}}{2 m}-\mu}{E_{k}}\right),
$$

with $u_{k}^{2}=1-v_{k}^{2}$. The chemical potential $\mu$ and the gap energy $\Delta$ are obtained by solving equations (8) and (9). Unfortunately, in the continuum limit, due to the choice of a contact potential, the gap equation diverges in the ultraviolet. This divergence is logarithmic in two dimensions and linear in three dimensions.

As discussed in [33], quite generally in two dimensions the bound-state energy $\epsilon_{B}$ exists for any value of the interaction strength $g$. For the contact potential the bound-state equation is

$$
-\frac{1}{g}=\frac{1}{V} \sum_{\mathbf{k}} \frac{1}{\frac{\hbar^{2} k^{2}}{2 m}+\epsilon_{B}},
$$

and then subtracting this equation from the gap equation [10, 11, 12], one obtains a regularized gap equation

$$
\sum_{\mathbf{k}}\left(\frac{1}{\frac{\hbar^{2} k^{2}}{2 m}+\epsilon_{B}}-\frac{1}{2 E_{k}}\right)=0 \text {. }
$$

In the two-dimensional continuum limit $\sum_{\mathbf{k}} \rightarrow$ $V /(2 \pi)^{2} \int d^{2} \mathbf{k} \rightarrow V /(2 \pi) \int k d k$, taking into account the functional dependence (11) of the amplitudes $u_{k}$ and $v_{k}$ on $\mu$ and $\Delta$, the Eq. (13) gives

$$
\epsilon_{B}=\Delta\left(\sqrt{1+\frac{\mu^{2}}{\Delta^{2}}}-\frac{\mu}{\Delta}\right)
$$

while the number equation (8) becomes

$$
n=\frac{N}{V}=\left(\frac{m}{2 \pi \hbar^{2}}\right) \Delta\left(\frac{\mu}{\Delta}+\sqrt{1+\frac{\mu^{2}}{\Delta^{2}}}\right) .
$$

These two equations are exactly those found in the appendix B of the paper of Marini, Pistolesi and Strinati [33]. We observe that, for a $2 \mathrm{D}$ inter-atomic potential described by a $2 \mathrm{D}$ circularly symmetric well of radius $R_{0}$ and depth $U_{0}$, the bound-state energy $\epsilon_{B}$ is given by $\epsilon_{B} \simeq \hbar^{2} /\left(2 m R_{0}^{2}\right) \exp \left(-2 \hbar^{2} /\left(m U_{0} R_{0}^{2}\right)\right)$ with $U_{0} R_{0}^{2} \rightarrow 0$ [30].

As previously stressed, several properties of ultra-cold Fermi gases have been investigated in the last few years by using the EBCS equations [13, 14]. Here we analyze the condensate fraction of fermionic pairs that is strictly related to the off-diagonal long-range order (ODLRO) 37] of the system. As shown by Yang 38], the BCS state guarantees the ODLRO of the Fermi gas, namely that, in the limit wherein both unprimed coordinates approach an infinite distance from the primed coordinates, the twobody density matrix factorizes as follows:

$$
\begin{array}{r}
\left\langle\hat{\psi}_{\uparrow}^{+}\left(\mathbf{r}_{1}^{\prime}\right) \hat{\psi}_{\downarrow}^{+}\left(\mathbf{r}_{2}^{\prime}\right) \hat{\psi}_{\downarrow}\left(\mathbf{r}_{1}\right) \hat{\psi}_{\uparrow}\left(\mathbf{r}_{2}\right)\right\rangle \\
=\left\langle\hat{\psi}_{\uparrow}^{+}\left(\mathbf{r}_{1}^{\prime}\right) \hat{\psi}_{\downarrow}^{+}\left(\mathbf{r}_{2}^{\prime}\right)\right\rangle\left\langle\hat{\psi}_{\downarrow}\left(\mathbf{r}_{1}\right) \hat{\psi}_{\uparrow}\left(\mathbf{r}_{2}\right)\right\rangle .
\end{array}
$$

The largest eigenvalue $N_{0}$ of the two-body density matrix (16) gives the number of Fermi pairs in the lowest state, i.e. the condensate number of Fermi pairs [11, 38, 39]. This number is given by

$$
N_{0}=\int d^{3} \mathbf{r}_{1} d^{3} \mathbf{r}_{2}\left|\left\langle\hat{\psi}_{\downarrow}\left(\mathbf{r}_{1}\right) \hat{\psi}_{\uparrow}\left(\mathbf{r}_{2}\right)\right\rangle\right|^{2},
$$

and it is straightforward to show [39] that

$$
N_{0}=\sum_{\mathbf{k}} u_{k}^{2} v_{k}^{2}
$$

In the two-dimensional continuum limit we find

$$
n_{0}=\frac{N_{0}}{V}=\frac{1}{4} \int \frac{d^{2} k}{2 \pi} \frac{\Delta^{2}}{\left(\frac{\hbar^{2} k^{2}}{2 m}-\mu\right)^{2}+\Delta^{2}},
$$


from which

$$
n_{0}=\frac{1}{4}\left(\frac{m}{2 \pi \hbar^{2}}\right) \Delta\left(\frac{\pi}{2}+\arctan \left(\frac{\mu}{\Delta}\right)\right) .
$$

Finally, by using Eq. (15) and Eq. (20) we obtain a remarkably simple formula for the condensed fraction

$$
\frac{n_{0}}{n}=\frac{1}{4} \frac{\frac{\pi}{2}+\arctan \left(\frac{\mu}{\Delta}\right)}{\frac{\mu}{\Delta}+\sqrt{1+\frac{\mu^{2}}{\Delta^{2}}}},
$$

This is the main result of the paper. Nicely, in Eq. (21) the condensate fraction depends only on the parameter $x_{0}=\mu / \Delta$. In the weakly-bound BCS regime $\left(x_{0} \gg 1\right)$ the condensed fraction $n_{0} / n$ goes to zero, while in the strongly-bound BEC regime $\left(x_{0} \ll-1\right)$ the condensed fraction $n_{0} / n$ goes to $1 / 2$, i.e. all the $N / 2$ Fermi pairs belong to the Bose-Einstein condensate.

In $2 \mathrm{D}$ the Fermi energy $\epsilon_{F}=\hbar^{2} k_{F}^{2} /(2 m)$ of a noninteracting Fermi gas is given by $\epsilon_{F}=\pi \hbar^{2} n / m$. It is convenient to express all relevant energies in terms of the Fermi energy $\epsilon_{F}$. In this way these scaled quantities depend only on the parameter $x_{0}$. In particular, we find

$$
\begin{gathered}
\frac{\epsilon_{B}}{\epsilon_{F}}=2 \frac{\sqrt{1+x_{0}^{2}}-x_{0}}{\sqrt{1+x_{0}^{2}}+x_{0}}, \\
\frac{\Delta}{\epsilon_{F}}=2\left(\sqrt{1+x_{0}^{2}}-x_{0}\right),
\end{gathered}
$$

and also

$$
\frac{\mu}{\epsilon_{F}}=2 x_{0}\left(\sqrt{1+x_{0}^{2}}-x_{0}\right) \text {. }
$$

All these quantities are parametrized by $x_{0}$. It is then quite easy to plot the scaled chemical potential $\mu / \epsilon_{F}$ and the scaled energy gap $\Delta / \epsilon_{F}$ as a function of the scaled bound-state energy $\epsilon_{B} / \epsilon_{F}$.

Fig. 1 shows that the scaled energy gap $\Delta / \epsilon_{F}$ (solid line) grows by increasing $\epsilon_{B} / \epsilon_{F}$ while the scaled chemical potential $\mu / \epsilon_{F}$ (dashed line) decreases. The chemical potential $\mu$ is zero when the bound-state energy $\epsilon_{B}$ is equal to $2 \epsilon_{F}$. For larger values of $\epsilon_{B}$ the chemical potential $\mu$ becomes negative. Physically, we can say that the value $2 \epsilon_{F}$ discriminates between the BCS regime of weekly-bound fermionic pairs $\left(0 \leq \epsilon_{B}<2 \epsilon_{F}\right.$ and $\left.\mu>0\right)$ and the BEC regime of strongly-bound fermionic pairs $\left(\epsilon_{B}>2 \epsilon_{F}\right.$ and $\left.\mu<0\right)$.

By using Eq. (21) and Eq. (22) we can also plot the condensate fraction $n_{0} / n$ as a function of the scaled bound-state energy $\epsilon_{B} / \epsilon_{F}$.

Fig. 2 shows the condensate fraction $n_{0} / n$ of Fermi pairs. The fraction is zero when the bound-state energy $\epsilon_{B}$ is zero. For small values of $\epsilon_{B} / \epsilon_{F}$ the condensed fraction has a very fast grow but then it reaches the asymptotic value $1 / 2$ very slowly. Note that at $\epsilon_{B} / \epsilon_{F}=2$ (where $\mu=x_{0}=0$ ) the condensate fraction is $\pi / 8 \simeq 0.39$.

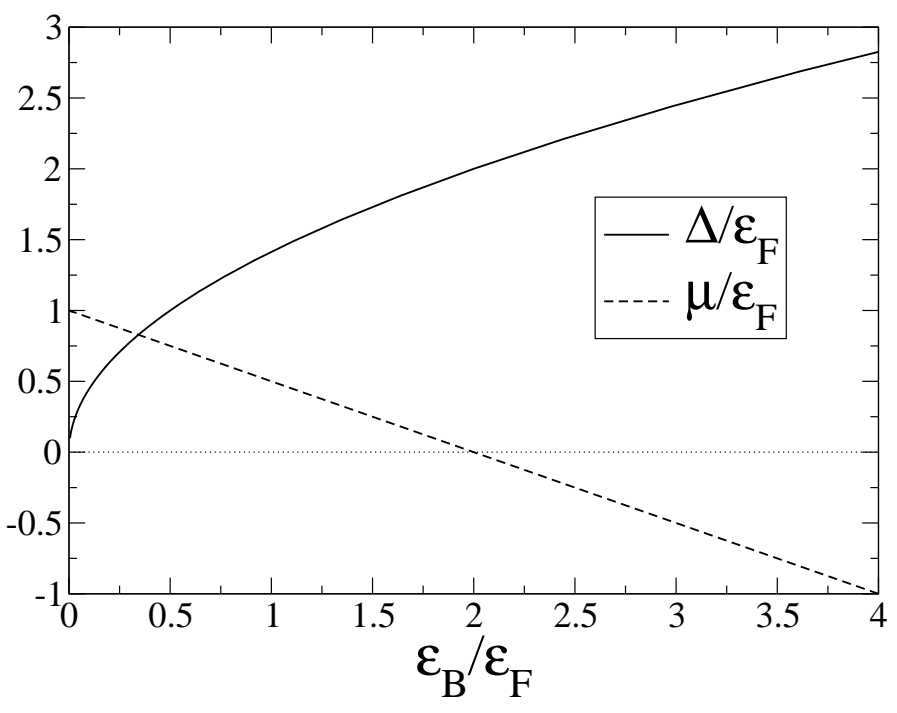

FIG. 1: Energy gap $\Delta$ (solid line) and chemical potential $\mu$ (dashed line) in the uniform two-component dilute 2D Fermi gas as a function of scaled bound-state energy $\epsilon_{B} / \epsilon_{F}$. The horizontal dotted line simply shows the zero.

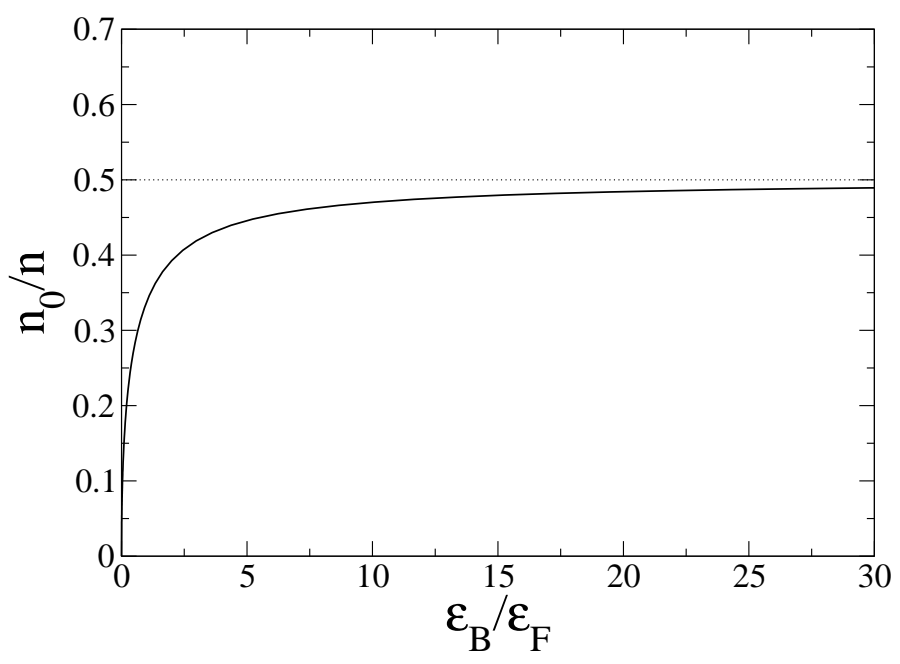

FIG. 2: Condensate fraction $n_{0} / n$ of Fermi pairs (solid line) in the uniform two-component dilute 2D Fermi gas as a function of scaled bound-state energy $\epsilon_{B} / \epsilon_{F}$. The horizontal dotted line shows the asymptotic value $n_{0} / n=1 / 2$.

To the sake of completeness, we observe that in the $3 \mathrm{D}$ case the condensate fraction can be expressed in terms of the dimensionless inverse interaction parameter $y=$ $\left(k_{F} a_{F}\right)^{-1}$, where $k_{F}$ is the Fermi wave vector of $3 \mathrm{D}$ noninteracting fermions and $a_{F}$ is the $3 \mathrm{D}$ s-wave scattering length of inter-atomic potential [19]. In this case, $y \in$ $(-\infty,+\infty)$ and only for $y>0$ there is the formation of a dimer with bound-state energy $\epsilon_{B} \simeq \hbar^{2} /\left(2 m a_{F}^{2}\right)$. The condensed fraction goes to zero for $y \rightarrow-\infty$ and to onehalf for $y \rightarrow+\infty$ [19].

In conclusion, by using the mean-field extended BCS theory and the concept of off-diagonal long-range order, 
that is the existence of a macroscopic eigenvalue of the two-body density matrix, we have obtained a remarkably simple formula for the condensate fraction of fermionic pairs in a uniform 2D Fermi gas. Contrary to the 3D case, in the 2D case a bound state appears immediately as the two-body attraction is introduced. As a consequence, the crossover from the BCS state of weakly-bound Cooper pairs to the BEC of strongly-bound dimers is induced by the increasing of the bound-state energy. We have show that the condensate fraction of Fermi pairs grows smoothly during the 2D BCS-BEC crossover, but only for a very large bound-state energy one gets a quasi complete condensation. It is important to stress that our predictions on the behavior of the condensed fraction in a $2 \mathrm{D}$ attractive Fermi gas can be surely compared with Monte Carlo calculations, as done in the $3 \mathrm{D}$ case [22]. On the other hand, it could be difficult to compare the theory with experiments. In fact, strictly 2D superfluid Fermi gases have not yet been achieved: a $2 \mathrm{D}$ configuration requires $n a_{H}^{2} \ll 1$, where $n$ is the $2 \mathrm{D}$ number density and $a_{H}$ is the characteristic length of a very strong harmonic confinement along one of the three axes [40]. In addition, in two-dimensions it could be more problematic than in three-dimensions to modify and trigger the bound-state energy [41]. Surely in the next future these issues will be faced and probably the obstacles will be overcomed.

The author thanks A. Parola and F. Toigo for useful discussions.
[1] M. Greiner, C.A. Regal, and D.S. Jin, Nature (London) 426, 537 (2003).

[2] C.A. Regal, M. Greiner, and D.S. Jin, Phys. Rev. Lett. 92, 040403 (2004).

[3] J. Kinast, S.L. Hemmer, M.E. Gehm, A. Turlapov, and J.E. Thomas, Phys. Rev. Lett. 92, 150402 (2004).

[4] M.W. Zwierlein et al., Phys. Rev. Lett. 92, 120403 (2004); M.W. Zwierlein, C.H. Schunck, C.A. Stan, S.M.F. Raupach, and W. Ketterle, Phys. Rev. Lett. 94, 180401 (2005).

[5] C. Chin et al., Science 305, 1128 (2004); M. Bartenstein et al., Phys. Rev. Lett. 92, 203201 (2004).

[6] G.M. Falco and H.T.C. Stoof, Phys. Rev. Lett. 92, 130401 (2004).

[7] A. V. Avdeenkov and J. L. Bohn, Phys. Rev. A 71, 023609 (2005).

[8] R.B. Diener and T.L. Ho, cond-mat/0404517.

[9] A. Perali, P. Pieri, and G.C. Strinati, cond-mat/0501631.

[10] D.M. Eagles, Phys. Rev. 186, 456 (1969).

[11] A.J. Leggett, Quantum Liquids. Bose Condensation and Cooper Pairing in Condensed-Matter Systems (Oxford Univ. Press, Oxford, 2006).

[12] P. Noziers and S. Schmitt-Rink, J. Low Temp. Phys. 59, 195 (1985).

[13] A. Perali, P. Pieri, and G.C. Strinati, Phys. Rev. A 68, 031601(R) (2003); A. Perali, P. Pieri, and G.C. Strinati, Phys. Rev. Lett. 93, 100404 (2004).

[14] H. Hu, A. Minguzzi, X.J. Liu, and M.P. Tosi, Phys. Rev. Lett. 93, 190403 (2004).

[15] N. Manini and L. Salasnich, Phys. Rev. A 71, 033625 (2005); G. Diana, N. Manini, and L. Salasnich, Phys. Rev. A 73, 065601 (2006); L. Salasnich and N. Manini, Laser Phys. 17, 169 (2007).

[16] G.E. Astrakharchik et al., Phys. Rev. Lett. 95, 030404 (2005); T.K. Ghosh and K. Machida, Phys. Rev. A 73, 025601 (2006); Y. Zhau and G. Huang, Phys. Rev. A 75 023611 (2007); A. Altmeyer et al., Phys. Rev. Lett. 98, 040401 (2007).

[17] C.A.R. Sa de Melo, M. Randeria, and J.R. Engelbrecht, Phys. Rev. Lett. 71, 3202 (1993).

[18] J.R. Engelbrecht, M. Randeria, and C.A.R. Sa de Melo, Phys. Rev. B 55, 15153 (1997).

[19] L. Salasnich, N. Manini, and A. Parola, Phys. Rev. A 72, 023621 (2005).
[20] G. Ortiz and J. Dukelsky, Phys. Rev. A 72, 043611 (2005).

[21] Y. Ohashi and A. Griffin, Phys. Rev. A 72, 063606 (2005).

[22] G. E. Astrakharchik, J. Boronat, J. Casulleras, and S. Giorgini, Phys. Rev. Lett. 95, 230405 (2005).

[23] J. Schneider and H. Wallis, Phys. Rev. A 571253 (1998).

[24] L. Salasnich, J. Math. Phys. 418016 (2000).

[25] L. Salasnich, B. Pozzi, A. Parola and L. Reatto, J. Phys. B: At. Mol. Opt. 333943 (2000).

[26] P. Vignolo and A. Minguzzi, Phys. Rev. A 67053601 (2003).

[27] A. Minguzzi, P. Vignolo, M.L. Chiofalo, and M.P. Tosi, Phys. Rev. A 64033605 (2001).

[28] K.K. Das, Phys. Rev. Lett. 90170403 (2003).

[29] L. Salasnich, S.K. Adhikari, and F. Toigo, Phys. Rev. A 75023616 (2007).

[30] L.D. Landau and E.M. Lifshitz, Quantum Mechanics. Non Relativistic Theory. Course of Theoretical Physics, Vol. 3 (Pergamon Press, New York, 1989).

[31] M. Randeria, J.M. Duan, and L.Y. Shieh, Phys. Rev. B 41, 327 (1990).

[32] Z. Nussinov and S. Nussinov, cond-mat/0410597.

[33] M. Marini, F. Pistolesi, and G.C. Strinati, Eur. Phys. J. B 1, 151 (1998).

[34] M. Rodriguez-Pinilla, Signatures of Superfluidity in Atomic Fermi Gases, Doctor of Technology Dissertation - ISBN 951-22-6854-X (Helsinki University of Technology, Helsinki, 2003).

[35] J. Bardeen, L.N. Cooper, and J.R. Schrieffer, Phys. Rev. 108, 1175 (1957).

[36] A.L. Fetter and J.D. Walecka, Quantum Theory of Many Particle Systems (Mc Graw Hill, New York, 1971).

[37] O. Penrose, Phil. Mag. 42, 1373 (1951); O. Penrose and L. Onsager, Phys. Rev. 104, 576 (1956).

[38] C.N. Yang, Rev. Mod. Phys. 34, 694 (1962).

[39] C.E. Campbell, in Condensed Matter Theories, vol. 12, 131 (Nova Science, New York, 1997).

[40] S.K. Adhikari and L. Salasnich, Phys. Rev. A 75, 053603 (2007).

[41] P. Naidon, E. Tiesinga, W.F. Mitchell, and P.S. Julienne, New J. Phys. 9, 19 (2007). 